\title{
Zinc Protoporphyrin IX Binds Heme Crystals to Inhibit the Process of Crystallization in Plasmodium falciparum
}

\author{
Jayasree K Iyer, Lirong Shi, Anuraj H Shankar ${ }^{1}$, And David J Sullivan Jr
}

The intraerythrocytic Plasmodium falciparum parasite converts most of host hemoglobin heme into a nontoxic heme crystal. Erythrocyte zinc protoporphyrin IX, normally present at $0.5 \mu \mathrm{M}$, which is a ratio of 1:40000 hemes, can elevate 10-fold in some of the anemias associated with malaria disease protection. This work examines a binding mechanism for zinc protoporphyrin IX inhibition of heme crystallization similar to the antimalarial quinolines. Zinc protoporphyrin IX neither forms crystals alone nor extends on preformed heme crystals. Inhibition of both seed heme crystal formation and crystal extension occurs with an inhibitory concentration (IC) $)_{50}$ of $5 \mu \mathrm{M}$. Field emission in-lens scanning electron microscopy depicts the transition and inhibition of heme monomer aggregates to heme crystals with and without seeding of preformed hemozoin templates. In vitro zinc protoporphyrin IX, like the quinolines, binds to heme crystals in a saturable, specific, pH, and time-dependent manner. The ratio at saturation is approximately 1 zinc protoporphyrin IX per 250 hemes of the crystal. Unlike the quinolines, zinc protoporphyrin IX binds measurably in the absence of heme. Isolated ring and trophozoite stage parasites have an elevated zinc protoporphyrin IX to heme ratio 6 to 10 times that in the erythrocyte cytosol, which also corresponds to elevated ratios found in heme crystals purified from Plasmodium parasites. This work implicates protection from malaria by a mechanism where elevated zinc protoporphyrin IX in anemic erythrocytes binds to heme crystals to inhibit further crystallization. In endemic malaria areas, severe iron deficiency anemia should be treated with antimalarials along with iron replenishment.

\section{INTRODUCTION}

During the erythrocytic stages of its infection, the malaria parasite degrades host hemoglobin to acquire essential amino acids $(1,2)$. Toxic free heme or iron protoporphyrin IX (FePPIX), a byproduct of this proteolysis, is not catabolized by heme oxygenase as it is in mammalian cells, but instead is converted into a unique FePPIX crystal called hemozoin $(3,4)$. Inhibition of this FePPIX crystallization accounts for the mechanism by which the antimalarial quinolines act (5), although controversy still exists over the exact mechanism of action of crystal inhibition after the quinolines 1st bind to FePPIX (6-9). Numerous studies have shown that the quinolines bind FePPIX noncovalently (10-14). An additional theory suggests the FePPIX-quinoline complex incorporates into a growing crystal face that blocks further crystal extension $(6,15-17)$. Indeed, this observation may explain why quinidine is a more potent in vitro crystallization inhibitor than chloroquine, despite having a lower FePPIX affinity (8).

Zinc protoporphyrin IX (ZnPPIX), present in normal erythrocytes at $0.5-\mu \mathrm{M}$ concentrations, can elevate above $5-\mu \mathrm{M}$ concentrations in erythrocyte anemias because of thalassemias, iron deficiency, hemolysis, or chronic inflammatory disease (18-20). When amounts of bioavailable iron in the reticulocyte are low, the ferrochelatase enzyme inserts zinc into protoporphyrin IX instead of iron, resulting in elevated ZnPPIX in the erythrocyte expressed as a ratio of $\mu$ mol ZnPPIX to mol of Fe PPIX exceeding the normal value of approximately $25 \mu \mathrm{mol} Z \mathrm{nPPIX}$ to $1 \mathrm{~mol}$ FePPIX (19). The
ZnPPIX resides in the FePPIX pockets of hemoglobin (19). Stoltzfus and coworkers have recently assessed iron status indicators in 611 well, non-febrile preschool children from Zanzibar living in an highly endemic malarious area (21). Amongst children in the lowest hemogolobin quartile, a negative malaria blood film correlated with a markedly higher mean ZnPPIX to FePPIX ratio above $250 \mu \mathrm{mol} Z \mathrm{ZnPPIX} / \mathrm{mol}$ FePPIX. This is interpreted as an important trend, but not conclusive in correlating an elevated ZnPPIX/FePPIX ratio with malaria protection.

Iron deficiency anemia affects over half of all young children and pregnant women in developing countries resulting in over 500 million cases of anemia worldwide (22). Endemic malaria and iron deficiency anemia coexist with mortality targeting young children and pregnant women $(21,23)$. Many but not all clinical studies of iron deficiency have also reported protection from severe malaria disease and exacerbation of malaria disease in individuals undergoing iron repletion (24). Interestingly, for some of the hemoglobinopathies and also iron deficiency anemia, malaria disease protection occurs without changing incidence of parasitemia, although the degree of parasitemia was not determined $(24,25)$.

Martiney and Cerami have already demonstrated ZnPPIX inhibition of FePPIX crystal formation in vitro and postulated a role for elevated ZnPPIX in $\beta$-thalassemic erythrocytes in protection from malaria (26). In vitro, nonmetal and noniron protoporphyrins have also been shown to inhibit the crystallization of FePPIX using, as a template for extension assays, FePPIX

The W Harry Feinstone Department of Molecular Microbiology and Immunology, Department of International Health, The Johns Hopkins University Bloomberg School of Public Health, Baltimore, MD.

INew address: Helen Keller International, Jalan Bungur Dalam 23A, Kemang, Jakarta, Indonesia. 
crystal derived from either from trophozoite lysates or from a chemical synthesis called $\beta$-hematin $(27,28)$. The binding affinity, near $5.6 \times 10^{5} / \mathrm{M}^{-1}$, of ZnPPIX to FePPIX by $\pi-\pi$ interactions has been reported to be similar to chloroquine $(28,29)$. The binding of a FePPIX-quinoline complex to preformed FePPIX crystals has been shown to be saturable and specific $(15,16)$.

In this report, we investigated the mechanism of ZnPPIX inhibition of heme crystallization by binding to crystals. We also measured the amount of ZnPPIX bound to FePPIX crystals isolated from $P$. falciparum-infected erythrocytes. A new qualitative method, field emission in-lens scanning electron microscopy (FEISEM), compares the morphology of FePPIX crystal formation and inhibition growth extension. These biochemical data add further evidence for elevated ZnPPIX in the anemic erythrocyte providing protection from severe malaria disease by heme crystal inhibition.

\section{MATERIALS AND METHODS}

\section{Materials}

Hemozoin from P. falciparum infected erythrocytes and synthetic $\beta$-hematin were isolated as described previously (16). ZnPPIX was obtained from Porphyrin Products Ltd (Logan, UT, USA). All other reagents were from Sigma (St. Louis, MO, USA) unless specified.

\section{FePPIX Crystal Assay}

Purified FePPIX crystals (from 5 to 100 nmol) were incubated with $50 \mathrm{nmol}$ of FePPIX chloride monomer overnight in $1 \mathrm{~mL}$ of 100 $\mathrm{mM}$ sodium acetate, $\mathrm{pH} 4.8$, in a $37^{\circ} \mathrm{C}$ water bath with different concentrations of ZnPPIX or drug. The next day, the samples were centrifuged at $10000 \times g$ after the addition of $100 \mu \mathrm{L}$ of wash buffer $-100 \mathrm{mM}$ sodium bicarbonate, $0.5 \%$ Triton X-100. The supernate was removed and the pellet suspended in wash buffer by sonication at the lowest setting and recentrifuged. This step ensures that unbound FePPIX and ZnPPIX are washed off of insoluble FePPIX crystals. The FePPIX crystal pellet is washed additionally with $0.5 \%$ Triton $X-100$. The final pellet is dissolved in $2 \%$ SDS and $20 \mathrm{mM}$ sodium hydroxide to measure protoporphyrin IX content by UV spectrophotometric analysis at 400 and $416 \mathrm{~nm}$ (Beckman DU 640 spectrophotometer). FePPIX and ZnPPIX were prepared fresh daily in dimethyl sulfoxide with an extinction coefficient $\varepsilon_{400}=100000 \mathrm{M}^{-1} \mathrm{~cm}^{-1}$ and $260000 \mathrm{M}^{-1} \mathrm{~cm}^{-1}$ respectively for FePPIX and ZnPPIX in SDS/ $20 \mathrm{mM}$ sodium hydroxide.

\section{ZnPPIX Determination}

The amount of ZnPPIX bound to the crystals was directly assayed by an ethyl acetate:acetic acid $\mathrm{HCl}$ extraction method, where $20 \mu \mathrm{L}$ of the sample was dissolved in $1 \mathrm{~mL}$ of 3:1 of ethyl acetate (OmniSolv Grade, EM Science, Gibbstown, NJ, USA) and acetic acid, which was centrifuged in glass tubes, and decanted into another similar tube. One milliliter of ethyl acetate and $1 \mathrm{~mL}$ of $3 \mathrm{~N}$ hydrochloric acid (high-performance liquid chromatography grade) was then added, the sample was vortexed briefly and centrifuged again, and the extracted ZnPPIX fluorescence was read in an optical fluorometer (Model 4-7349 American Instrument Co, Silver Spring, MD, USA) (30). Control standards of known concentration of FePPIX and ZnPPIX were also set up and assayed to correlate intensity of fluorescence to actual amounts of ZnPPIX bound. The lower limit of detection is approximately 20 pmol of ZnPPIX. Alternatively, 15 to $20 \mu \mathrm{L}$ of sample were placed onto a coverslip and the ZnPPIX to FePPIX ratio was read directly on a hematofluorometer (AVIV Biomedicals). The lower limit sensitivity is improved with this ratio determination. For all determinations, the purified crystals of FePPIX with ZnPPIX bound were treated with $20 \mathrm{mM}$ sodium hydroxide to decrystallize hemozoin. FePPIX crystals or hemozoin absorb substantially less than monomeric FePPIX at $400 \mathrm{~nm}$, which will artificially increase the ZnPPIX value in relation to FePPIX in intact crystals (31).

\section{Binding Competition Assay}

We incubated $10 \mathrm{nmol}$ of ZnPPIX and $10 \mathrm{nmol}$ of FePPIX crystals with and without $10 \mathrm{nmol}$ of FePPIX monomer and $10 \mathrm{nmol}$ of drugs in $1 \mathrm{~mL}$ of $100 \mathrm{mM}$ sodium acetate overnight at $37^{\circ} \mathrm{C}$. The samples were purified by Triton X-100 and bicarbonate washes as above with quantification of FePPIX and ZnPPIX by visible spectroscopy and the ethyl acetate method. SEM was calculated for all assays on triplicate samples.

\section{Parasite-associated ZnPPIX}

Parasites were cultured in human erythrocytes and synchronized by sorbitol lysis $(32,33)$. Ring, trophozoite, or schizont stage parasite cultures were harvested by saponin lysis (34). A ZnPPIX to FePPIX ratio was determined on the AVIV hematofluorometer. An additional trophozoite pellet was processed on a $60 \%$ sucrose cushion (15). A separate parasite harvest was processed for hemozoin isolation (3). The parasite pellet was washed several times in $2 \%$ SDS and treated with $2 \mathrm{mg} / \mathrm{mL}$ proteinase $\mathrm{K}$, washed again with SDS, and treated with $6 \mathrm{M}$ urea. Samples were taken at every wash step and ratio of ZnPPIX to FePPIX was determined on the AVIV hematofluorometer.

\section{Field Emission In-lens Scanning Electron Microscopy (FEISEM)}

Prior to sample application, $5 \times 5 \mathrm{~mm}$ silica chips (Ted Pella, cat\#16008, Redding, CA, USA) were cleaned with ethanol, coated with poly-lysine, and then rinsed 3 times with distilled water. Incubations of 1 to 3 nmol FePPIX crystals with or without monomers were applied onto chips in distilled water or the sodium acetate buffer. After centrifugation at $3000 \times g$ for 10 min, FePPIX crystals formed a thin layer on the chip surface. The sodium acetate buffer was diluted 50 fold by addition of distilled water and recentrifuged. Chips were then stained with $2 \%$ uranyl acetate for 30 to $60 \mathrm{~min}$. The chips and crystals or monomers coating them were continuously in aqueous solution until after uranyl acetate staining. The procedure was next modified from the previous report (35) to eliminate ethanol washes that dissolve protoporphyrin IX monomers before a critical point drying. Normally, after 1 quick rinse with 50\% ethanol, chips are rinsed consecutively in 50\%,70\%, $90 \%$ ethanol for $3 \mathrm{~min}$ each, followed by 3 rinses in 100\% ethanol for $3 \mathrm{~min}$ each before critical point drying for 1 to $2 \mathrm{~h}$. Chips processed either with ethanol washes and critical point drying or without ethanol were coated with chromium. Silica chips loaded with samples were ready for FEISEM on the LEO 1550 instrument. 


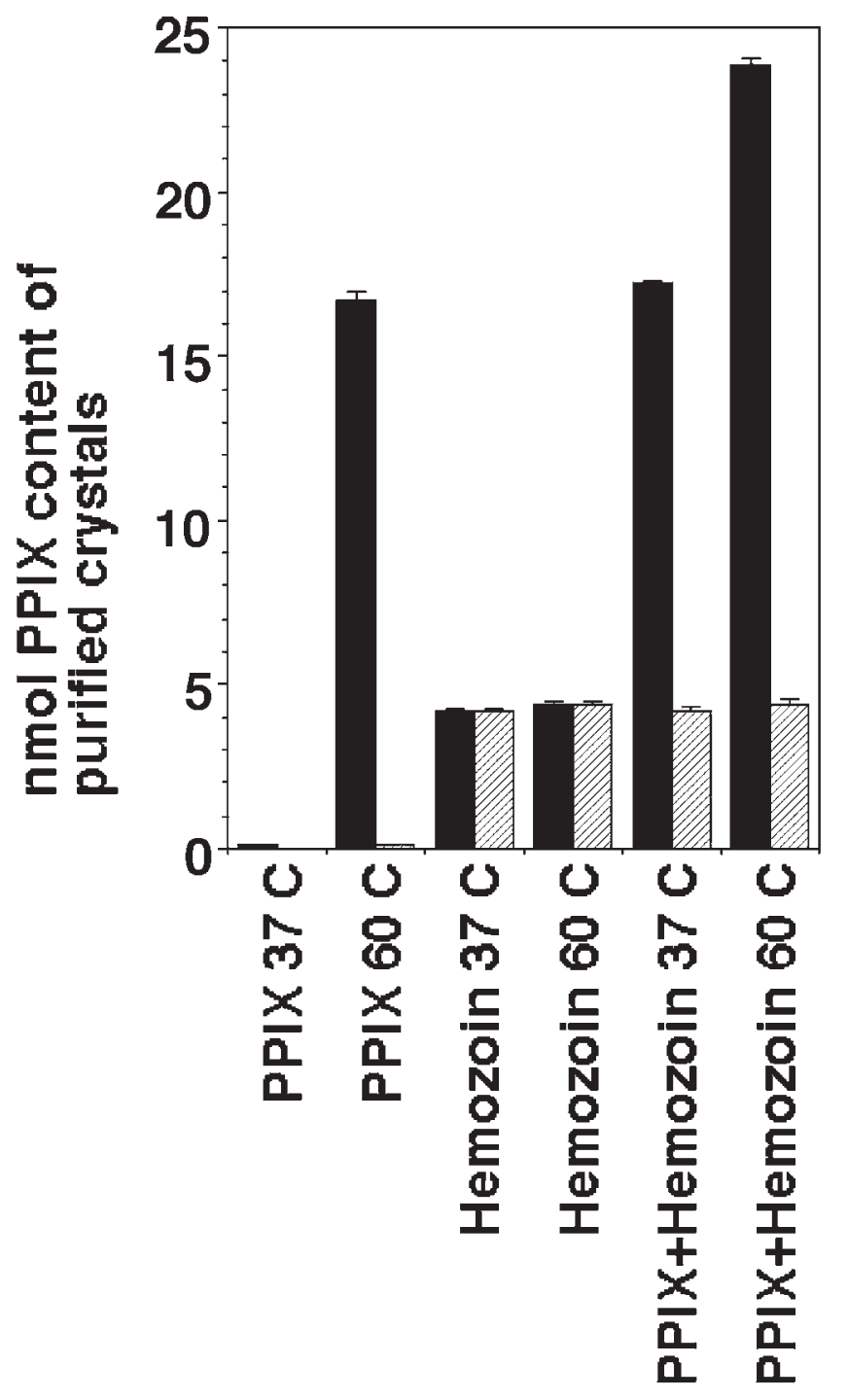

Figure 1. Crystal formation with FePPIX or ZnPPIX. Twenty-five nanomoles of FePPIX (solid bars) or ZnPPIX (hatched bars) were incubated overnight in $500 \mu \mathrm{L}$ of $100 \mathrm{mM}$ sodium acetate, $\mathrm{pH} 4.8$, at $37^{\circ} \mathrm{C}$ and $60^{\circ} \mathrm{C}$ with and without $5 \mathrm{nmol}$ hemozoin. The incubation product was processed to purify away unincorporated protoporphyrin species, and the crystal content remaining was quantified. Only FePPIX alone at $60^{\circ} \mathrm{C}$ or with hemozoin at $37^{\circ} \mathrm{C}$ and $60^{\circ} \mathrm{C}$ made measurable crystals. SEM is derived from triplicate samples.

\section{RESULTS}

\section{ZnPPIX Does Not Measurably Form or Extend FePPIX Crystals}

The yields of purified heme crystal conversion from monomer in acidic overnight incubations of $50 \mu \mathrm{M}$ FePPIX shows a temperature dependence (Figure 1 ). At $37^{\circ} \mathrm{C}$, less than $1 \%$ of substrate converts to crystals for FePPIX, whereas at $60^{\circ} \mathrm{C}$, approximately $70 \%$ of starting substrate converts to crystals for FePPIX. For similar $50 \mu \mathrm{M}$ ZnPPIX incubations, no measurable crystal conversion is detected at either temperature. The crystal conversion yield increases after addition of $5 \mathrm{nmol}$ of purified
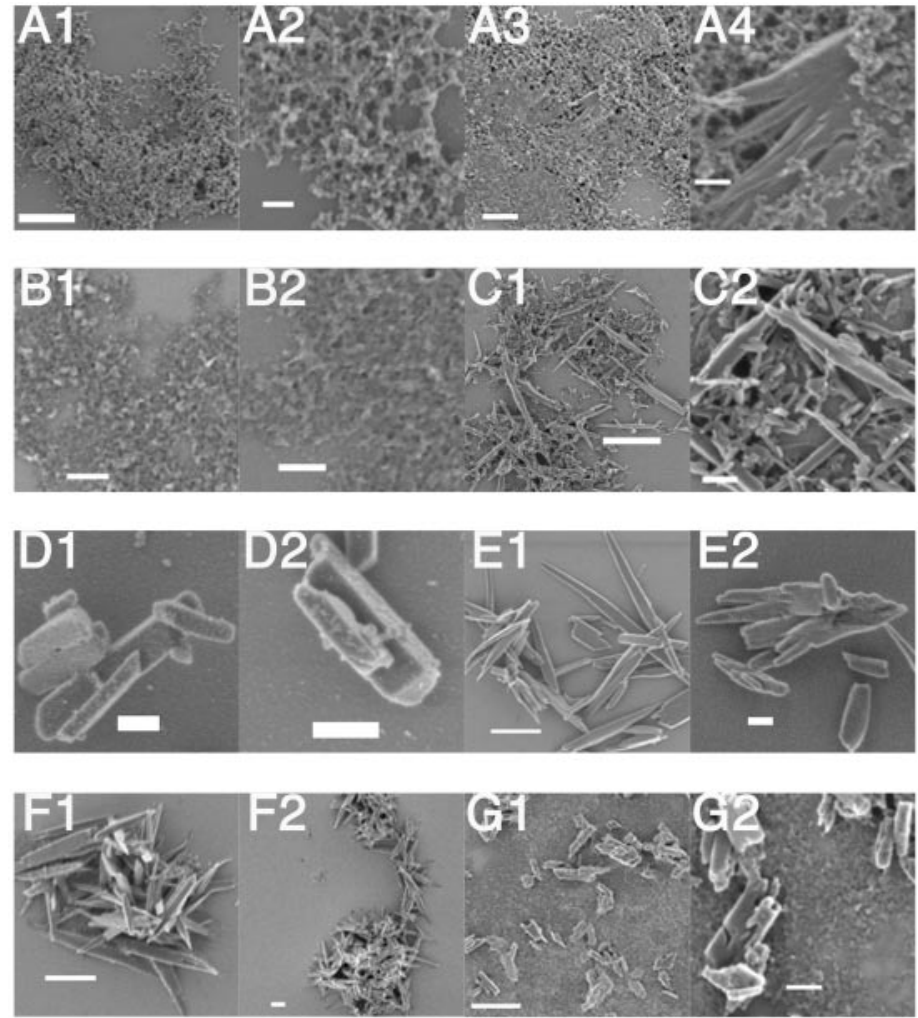

Figure 2. FEISEM images of crystal formation and extension. The incubation products from Figure 1 were deposited onto a silica chip without ethanol washes or drying so that they would more closely represent solution morphology of the metal porphyrins. A: Incubations of $50 \mu \mathrm{M}$ FePPIX at $37^{\circ} \mathrm{C}$ overnight remain $99 \%$ in moss-like aggregated state in $\mathrm{Al}$ and $\mathrm{A} 2$ and are seen with a rare seed crystal in A3 and A4. B: ZnPPIX at $37^{\circ} \mathrm{C}$ shows no seed crystals. C: When the temperature is raised to $60^{\circ} \mathrm{C}$, all of FePPIX monomer changes to long, thin, tapered crystals with no mossy aggregates seen. D: Extensively purified $P$. falciparum hemozoin has regular cuboidal smooth surfaces of about $100 \mathrm{~nm} \times 100 \mathrm{~nm}$ $\times 300 \mathrm{~nm}$. E: Extensively purified $\beta$-hematin is spiculated with a greater size range. F: Incubations of seeded hemozoin with $50 \mu \mathrm{M}$ FePPIX monomer show a spiculated appearance that resembles $\beta$-hematin more than the original $P$. falciparum hemozoin. G: ZnPPIX at both $37^{\circ} \mathrm{C}$ and $60^{\circ} \mathrm{C}$ shows no addition to preformed crystals. The size bar is $200 \mathrm{~nm}$ except for images $\mathrm{Al}, \mathrm{A} 3, \mathrm{Cl}, \mathrm{El}, \mathrm{Fl}, \mathrm{F2}$, and $\mathrm{Gl}$ where it is 1 micron.

preformed P. falciparum hemozoin seed crystals to the $50 \mu \mathrm{M}$ FePPIX substrate to about $50 \%$ and $80 \%$ of substrate at $37^{\circ} \mathrm{C}$ and $60^{\circ} \mathrm{C}$, respectively. In contrast, addition of hemozoin seed crystals to $50 \mu \mathrm{M}$ ZnPPIX does not measurably extend the preformed FePPIX crystals.

FEISEM qualitatively evaluates the morphology of the above experiments shown in Figure 1 in greater detail. The FEISEM imaging protocol was adapted to a fully aqueous deposition and metal coating, which to a degree partially preserves noncrystal heme aggregate morphology by eliminating ethanol washes and critical point drying. The amount of FEISEM distortion of the monomer or mu-oxy dimer that appears as moss-like aggregates is not known, yet crystals can easily be distinguished from noncrystal aggregates. Almost all FEISEM images of incubations with $50 \mu \mathrm{M}$ FePPIX at $37^{\circ} \mathrm{C}$ demonstrate no visible crystal formation (Figure 2A1 and 2A2). However in rare images, formation of a 

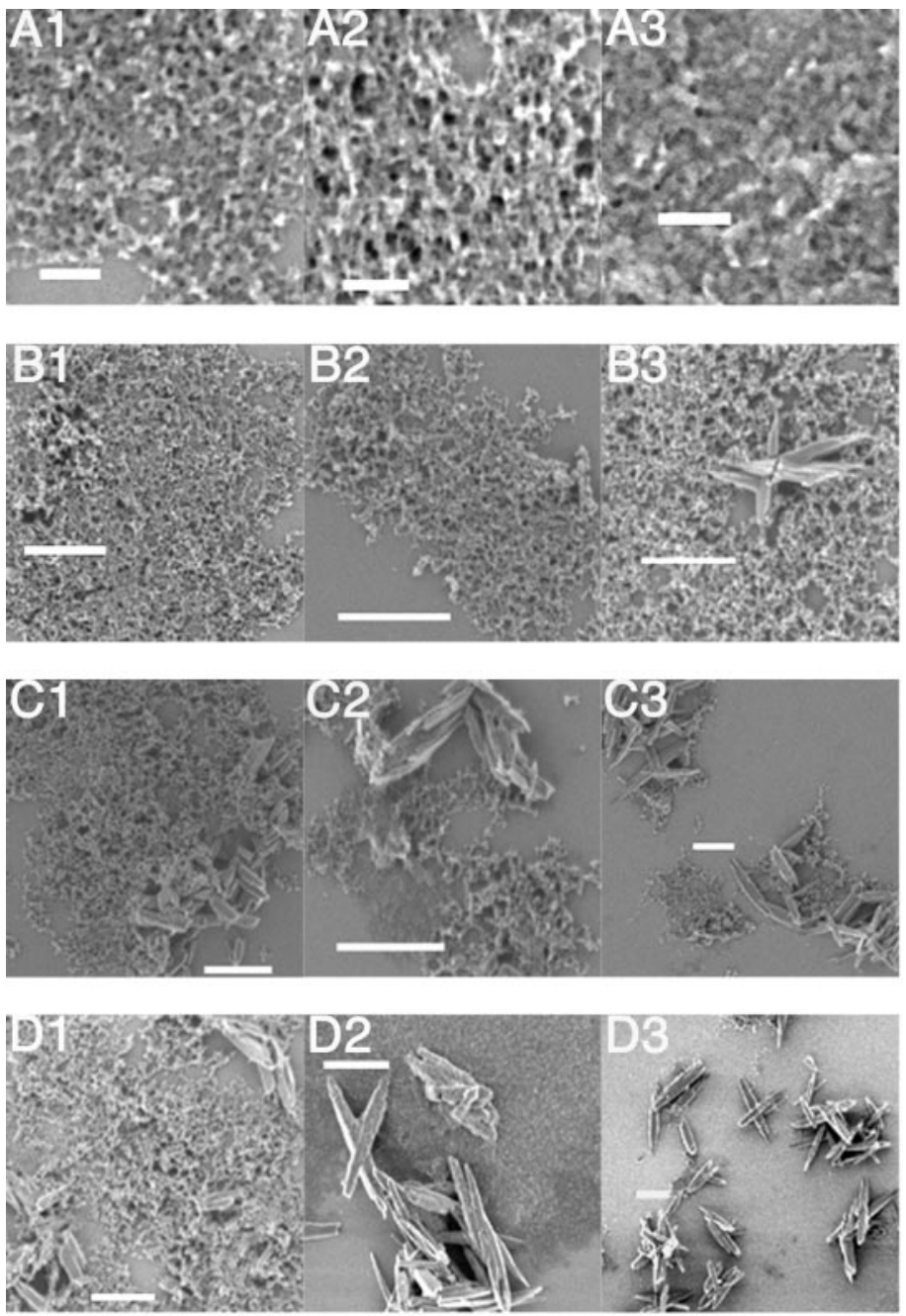

Figure 3. FEISEM images of $10 \mu \mathrm{M}$ PPIX or drugs with FePPIX with and without hemozoin. A: $10 \mu \mathrm{M}$ ZnPPIX, FePPIX, and chloroquine were mixed in solution with $100 \mathrm{mM}$ sodium acetate, $\mathrm{pH} 4.8$, vortexed, and spun onto the silica chips for FEISEM. A1: $10 \mu \mathrm{M}$ FePPIX and chloroquine. A2: $10 \mu \mathrm{M}$ ZnPPIX and FePPIX. A3: $10 \mu \mathrm{M} Z \mathrm{ZnPPIX}$ and chloroquine. B: Overnight incubations of $10 \mu \mathrm{M}$ chloroquine, ZnPPIX, or artemisinin (B1, B2, B3, respectively) with $50 \mu \mathrm{M}$ FePPIX showing only seed crystals with artemisinin. C and D: Incubations of $50 \mu \mathrm{M}$ FePPIX with $10 \mu \mathrm{M}$ chloroquine, ZnPPIX, artemisinin or no drug and the addition of $5 \mathrm{nmol}$ seed crystals of hemozoin at $0 \mathrm{~h}, 12 \mathrm{~h}$, and $24 \mathrm{~h}$. Cl: Time $0 \mathrm{~h}$ for chloroquine, hemozoin, and $50 \mu \mathrm{M}$ FePPIX. Time $0 \mathrm{~h}$ is identical for FePPIX and hemozoin (not shown). C2: $12 \mathrm{~h}$ incubation of ZnPPIX, hemozoin, and $50 \mu \mathrm{M}$ FePPIX. C3: $12 \mathrm{~h}$ incubation of hemozoin and $50 \mathrm{\mu M}$ FePPIX. Incubation of artemisinin, hemozoin, and FePPIX is identical to C3 (not shown). D1: $24 \mathrm{~h}$ incubation of chloroquine, hemozoin, and $50 \mu \mathrm{M}$ FePPIX showing no extension of crystal. The 12-h time point is identical (not shown). D2: 24-h incubations of ZnPPIX, hemozoin, and $50 \mu \mathrm{M}$ FePPIX showing some spicule crystal extension, but largely monomer aggregates. D3: 24-h incubations of artemisinin, hemozoin, and $50 \mu \mathrm{M}$ FePPIX showing almost total conversion of FePPIX substrate into FePPIX crystals. The size bar is $200 \mathrm{~nm}$ in A and 1 micron in B, C, and D.

seed crystal is seen amidst aggregated uncrystallized FePPIX (see Figure 2A3 and 2A4). The purified crystal yield remains less than $1 \%$ starting heme substrate under these conditions, even with the rare small FePPIX crystals seen. ZnPPIX at all time points and temperatures remains aggregated with no nidus of crystal formation detected despite scanning many fields (see Figure 2B).
Heme crystals from Plasmodium parasites, historically termed "hemozoin", are structurally identical to synthetic $\beta$-hematin made in vitro $(3,4)$. Both are $\beta$-hematins containing a reciprocal coordinate bond of the central iron to adjacent oxygen of the heme side chain propionate carboxylate group. The head-to-tail Fe1-O41 dimer has been defined by powder diffraction data (4). Synthetic heme crystals of $\beta$-hematin can vary in microscopic morphology $(36,37)$, but biochemically are similar to parasite hemozoin. Here we show that FEISEM analysis of unpurified $\beta$-hematin product at $60^{\circ} \mathrm{C}$ shows little moss-like aggregates, but large quantities of long thin tapered heme crystals of varying size (see Figure 2C). In contrast, purified P. falciparum hemozoin crystals have rectangular dimensions and smooth surfaces (see Figure 2D). Purified $\beta$-hematin prepared by the Egan method of heating $\mathrm{mM}$ amounts of heme in concentrated Molar solutions of acetic acid also has long thin tapering morphology, but less of the smaller crystals are recovered (see Figure 2E). Seeding the crystallization process with preformed hemozoin crystals accelerates the monomer to crystal conversion yield. The incubation mixture of preformed hemozoin FePPIX crystals from parasites with $50 \mu \mathrm{M}$ FePPIX at both temperatures depicts a long, thin, tapered extension with the resultant product morphology more typical of $\beta$-hematin rather than the more cuboidal $P$. falciparum hemozoin (see Figure 2F). In contrast to FePPIX, $50 \mu \mathrm{M}$ ZnPPIX alone as substrate incubated with hemozoin seed crystals shows no discernible addition of ZnPPIX to preformed crystals (see Figure 2G). A moss-like aggregate of unincorporated ZnPPIX is seen covering some of the preformed hemozoin crystals. Buller and colleagues have modeled the theoretical growth of heme crystals with discussion of a fast-growing dimension that may produce the long, thin, tapered crystals indicative of rapid in vitro growth. Conditions for growth in a test tube have not yet perfectly replicated the crystal morphology of the parasite, which may be more controlled. However, the biochemical characteristics of in vitro formation and inhibition approximate parasite biology.

\section{ZnPPIX Inhibits Heme Crystal Extension}

Spectroscopy has previously suggested $\pi-\pi$ porphyrin-porphyrin ring interaction of ZnPPIX with FePPIX is similar to binding of many quinolines. FEISEM imaging of $10 \mu \mathrm{M}$ solutions of ZnPPIX plus FePPIX were compared with chloroquine plus FePPIX and chloroquine plus ZnPPIX in $100 \mathrm{mM}$ sodium acetate, $\mathrm{pH} 4.8$ (Figure 3A). The aggregated porphyrins were similar in appearance. At these concentrations, chloroquine does not deflect downward the optical density of the ZnPPIX indicating minimal to no interactions with this quinoline (data not shown). In contrast, the optical density of FePPIX is deflected downward by chloroquine. The artemisinin class of antimalarials, sesquiterpene lactones, also bind heme, but do not inhibit heme crystallization. Instead, the artemisinin endoperoxide bridge after interacting with free iron produces an oxygen radical essential for antimalarial action. This drug has been shown to inhibit the PfATPase calcium pump and not accumulate in the digestive vacuole that chloroquine targets (38). Incubations of the heme binding drugs chloroquine $(10 \mu \mathrm{M}), \mathrm{ZnPPIX}$ and artemisinin with $50 \mu \mathrm{M}$ FePPIX for $24 \mathrm{~h}$ show no nidus of FePPIX crystal formation with chloroquine or ZnPPIX 
A

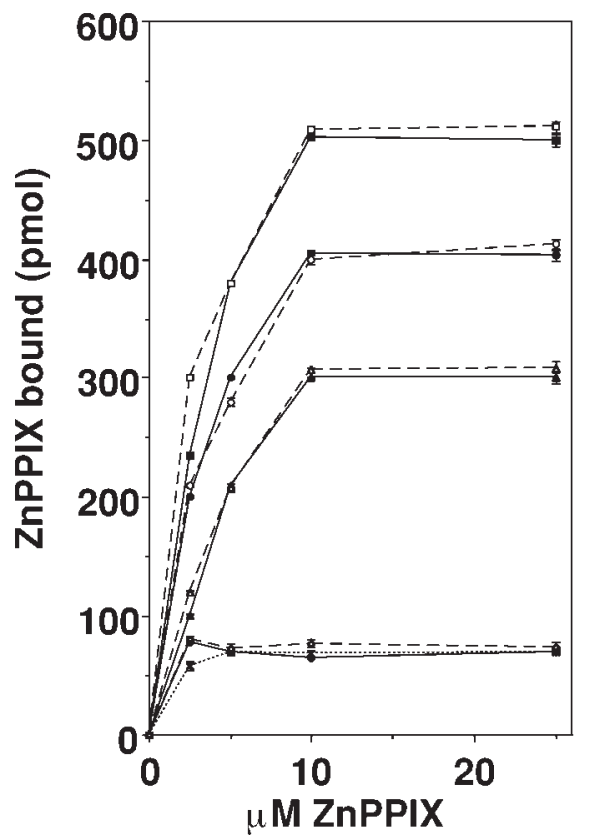

B

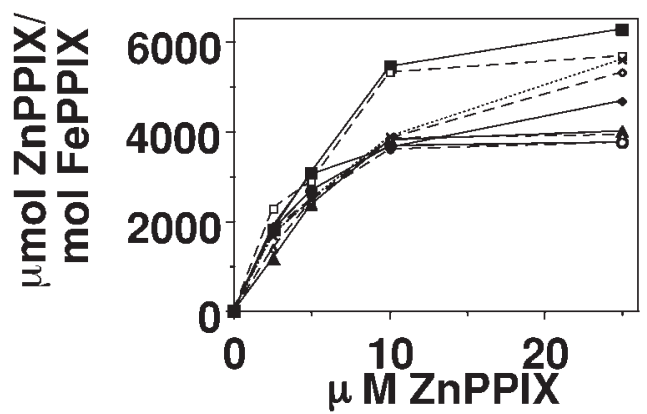

Figure 4. Saturable ZnPPIX binding to FePPIX crystals. A: ZnPPIX binding during a FePPIX crystal extension assay or directly to crystal rises with an increase in the amount of preformed FePPIX crystals used to seed the reaction. $100 \mathrm{nmol}(\boldsymbol{\square}), 75 \mathrm{nmol}(\mathbf{\bullet}), 50 \mathrm{nmol}(\mathbf{\Delta})$, and $10 \mathrm{nmol}(\bullet)$ of $\beta$-hematin or $10 \mathrm{nmol}$ of parasite hemozoin $(x)$ by FePPIX content was incubated with (filled symbols, solid lines) or without (empty symbols, dashed lines) $50 \mathrm{nmol}$ FePPIX substrate and increasing concentrations of ZnPPIX in $1 \mathrm{~mL}$ of $100 \mathrm{mM}$ sodium acetate, $\mathrm{pH} 4.8$, at $37^{\circ} \mathrm{C}$ overnight. The absolute amount determined by the ethyl acetate method of ZnPPIX bound after crystal purification was similar with or without the addition of FePPIX substrate. B: Molar ratios of ZnPPIX:FePPIX in purified FePPIX crystals saturated at 3000 to $6000 \mu \mathrm{mol} Z \mathrm{nPPIX} / \mathrm{mol}$ FePPIX. Picomoles of bound ZnPPIX in A are divided by nmol FePPIX content of recovered crystals. The symbols are the same as in A. SEM is calculated on triplicate samples.

(see Figure 3B1 and 3B2). However, incubations of artemisinin and FePPIX result in rare, small seed crystals (see Figure 3B3) similar to that of FePPIX alone, seen in Figure 2A3 and 2A4.

Chloroquine and ZnPPIX have previously been shown to have similar inhibitory concentrations to inhibit $50 \%$ of radioac- tive heme incorporation into preformed hemozoin (26). The heme crystal assay here uses significantly lower FePPIX concentrations and seed crystals of purified hemozoin. Both ZnPPIX and chloroquine inhibit FePPIX crystal extension with an inhibitory concentration of approximately 3 to $5 \mu \mathrm{M}$ for $50 \%$ of the monomer to crystal conversion yield. Again FEISEM is employed to visualize at $12 \mathrm{~h}$ and $24 \mathrm{~h}$ the amount of crystal formation (see Figure 3C). At time 0, no extension products are visualized for all incubations with FePPIX, chloroquine and hemozoin as a representative sample (see Figure 3C1). The lower right corner illustrates preformed hemozoin mixed in with noncrystalline aggregated heme. The incubations of $10 \mu \mathrm{M}$ ZnPPIX with $50 \mu \mathrm{M}$ FePPIX plus hemozoin template show most of the substrate to be aggregated at $12 \mathrm{~h}$ (see Figure 3C2). After $12 \mathrm{~h}$, substantially more of the FePPIX aggregates have converted to heme crystals in the reaction with no drug inhibition (see Figure 3C3). At $24 \mathrm{~h}$, chloroquine still prevented any crystal growth (see Figure 3D1). The crystals evident at the corners are the preformed crystals that show minimal to no extension. Similar to chloroquine, ZnPPIX has still prevented most of the crystal extension (see Figure 3D2) with little change from the 12-h time point. In contrast, artemisinin, which also binds FePPIX, shows no inhibition of crystal growth (see Figure 3D3).

\section{ZnPPIX Binds to FePPIX Crystals During Extension}

Because ZnPPIX is naturally fluorescent, we assay the amount of ZnPPIX association to the growing FePPIX crystals in a similar crystal extension assay to that used for the quinoline antimalarials (15). Using $10 \mathrm{nmol}$ of FePPIX crystals as a seed template either purified from parasites, or from synthetic $\beta$-hematin, approximately $70 \mathrm{pmol}$ of $\mathrm{ZnPPIX}$ binds at saturation (Figure 4). Increasing the quantity of $\beta$-hematin FePPIX crystals to 50,75 , and $100 \mathrm{nmol}$, increases the amount of ZnPPIX bound at saturation. The more available synthetic $\beta$-hematin was used for the larger amounts of heme crystal, because biochemically it has almost identical characteristics to hemozoin measured by ZnPPIX binding with 10 nmol FePPIX crystals, as well as drug inhibition and susceptibility to hydrogen peroxide degradation $(35,37)$. Control incubations with ZnPPIX alone or ZnPPIX plus 100 nmol of FePPIX monomer did not pellet detectable ZnPPIX in this assay (data not shown). Unlike the antimalarial quinolines that require the addition of FePPIX monomers for measurable binding to FePPIX crystals, the addition or deletion of $50 \mu \mathrm{M}$ FePPIX did not affect ZnPPIX saturation levels. A calculated $\mu \mathrm{mol}$ ZnPPIX to mol FePPIX ratio levels off at 3000 to 6000, for saturating amounts of ZnPPIX despite increasing amounts of FePPIX crystal template or deleting FePPIX monomer (see Figure 4B). This indicates that ZnPPIX is binding to limited sites of the FePPIX crystals at a ratio of 1 ZnPPIX per 167 to 333 FePPIX molecules for preformed crystal templates. Similar to FePPIX extension, ZnPPIX binding to crystal template is at its highest at low $\mathrm{pH}$ of 4.0 decreasing to $10 \%$ binding by $\mathrm{pH} 6.0$ in both MES and sodium acetate buffers (data not shown). A time course assay shows no measurable ZnPPIX bound to crystals at time 0 with increasing amounts measured up to a maximum at $16 \mathrm{~h}$ (data not shown). 


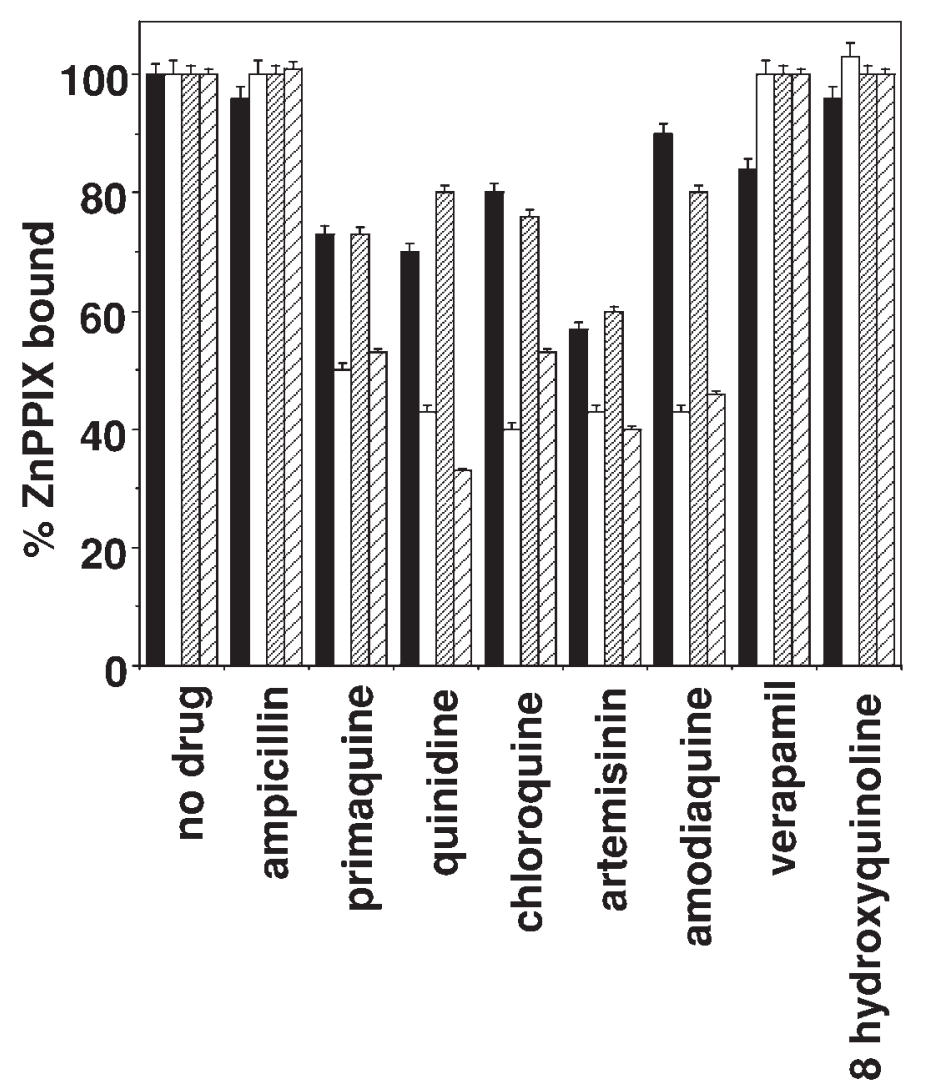

Figure 5. Antimalarial quinoline competition of ZnPPIX binding to crystals. ZnPPIX (10 nmol) and drug (10 nmol) were incubated with $10 \mathrm{nmol}$

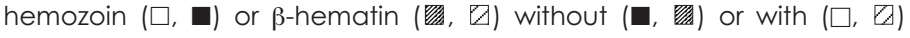
$10 \mathrm{nmol}$ FePPIX, overnight in $1 \mathrm{~mL}$ of $100 \mathrm{mM}$ sodium acetate, $\mathrm{pH}$ 4.8. The percent of ZnPPIX binding by the ethyl acetate method to recovered FePPIX crystal was measured. SEM is calculated on triplicate samples.

\section{Quinolines Compete for ZnPPIX Binding to FePPIX Crystals}

To investigate if specific antimalarial quinolines would compete for ZnPPIX binding, $10 \mu \mathrm{M}$ drug concentrations are coincubated with $10 \mu \mathrm{M}$ ZnPPIX in a FePPIX crystal binding assay without and with $10 \mu \mathrm{M}$ FePPIX substrate. This binding assay differs from the extension assay because the lower concentration of FePPIX substrate results in no measurable increase in heme crystal (16). Active quinoline antimalarials incubated without FePPIX substrate compete with the ZnPPIX binding to both parasite hemozoin and $\beta$ hematin in the range of $20 \%$ to $40 \%$ (Figure 5). Verapamil, 8 hydroxyquinoline, and ampicillin do not compete. The metal chelators such as ferrozine, desferoxime, neocuprione, EDTA, and EGTA also do not compete for ZnPPIX binding (data not shown). Interestingly adding $10 \mu \mathrm{M}$ FePPIX increases the amount of competition by the quinolines to $50 \%$ to $60 \%$. Five $\mu \mathrm{M}$ of ZnPPIX also competes $82 \%$ of ${ }^{3} \mathrm{H}$-chloroquine and $57 \%$ of ${ }^{3} \mathrm{H}$-quinidine binding to FePPIX crystal in an similar radioactive binding assay reported previously to assess quinoline binding to hemozoin (data not shown) (16). This suggests that ZnPPIX binds to the same sites on

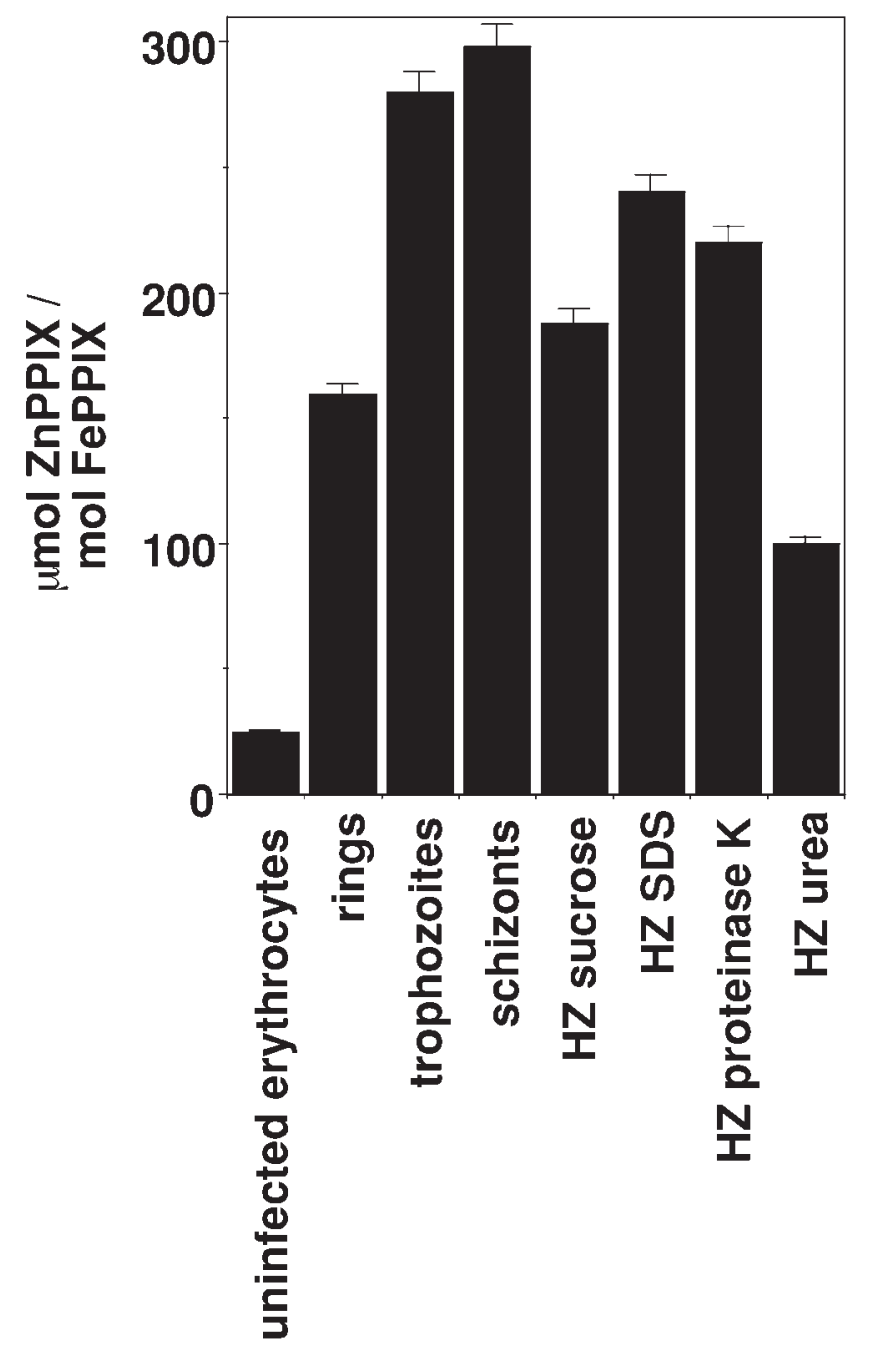

Figure 6. Elevated ratio of ZnPPIX to FePPIX in isolated parasite hemozoin. Parasites (3D7 strain of $P$. falciparum) were cultured in human erythrocytes and a saponin lysis purification was done at the stages of rings, trophozoites, and schizonts with greater than $90 \%$ synchrony. Trophozoite stages were also sonicated and placed on $60 \%$ sucrose cushions for ultracentrifugations to purify hemozoin ( $\mathrm{HZ}$ sucrose). This hemozoin was washed with SDS (HZ SDS), treated with Proteinase K (HZ proteinase $\mathrm{K}$ ), and urea ( $\mathrm{HZ}$ urea). ZnPPIX:FePPIX ratios by the AVIV hematofluorometer were measured after treatment with $20 \mathrm{mM}$ sodium hydroxide to decrystallize hemozoin.

the FePPIX crystals as the active quinoline antimalarials and that ZnPPIX binds in the absence of free FePPIX.

\section{ZnPPIX Copurifies with FePPIX Crystals Within the}

\section{Parasite}

An important validation of ZnPPIX inhibition of FePPIX crystallization is demonstration of increased ZnPPIX to FePPIX ratios in isolated parasites and more specifically in purified FePPIX crystals (Figure 6). A synchronized population of parasites shows increasing ratios of ZnPPIX to FePPIX in saponin-lysed purified parasite pellets as more FePPIX crystal is made. A sucrose cushion purification that separates hemozoin from membranes and solu- 
ble proteins shows a 10-fold elevation of ZnPPIX to FePPIX in hemozoin compared with ratios in normal erythrocytes. A more rigorous standard laboratory purification of hemozoin from parasite material involves washes with the detergent, SDS, followed by proteinase treatment and urea denaturation and further SDS and water washes $(3,37)$. Radioactive chloroquine and quinidine are stripped from parasite hemozoin with this purification (data not shown). However the ZnPPIX to FePPIX ratio remains elevated after SDS and proteinase K treatment, but decreases to half the ratio value with urea treatment.

If hemozoin with ZnPPIX bound that is recovered from parasites cultured in normal erythrocytes or hemozoin with ZnPPIX bound in vitro (incubations with 10 or $50 \mu \mathrm{M}$ ZnPPIX washed or diluted tenfold) is used to seed the in vitro FePPIX extension assay, less than $15 \%$ inhibition is seen. Chloroquine and quinidine also give the same minimal inhibition result.

\section{DISCUSSION}

This work demonstrates a role for the naturally occurring ZnPPIX in erythrocytes to inhibit FePPIX crystallization in Plasmodium by binding to the surface of FePPIX crystals. Here we demonstrate that ZnPPIX binding to FePPIX crystals is saturable and specific similar to the quinoline binding and inhibition. Unlike the quinolines, free heme is not a requirement for measurable ZnPPIX binding to crystals. Ring stage parasites also begin to make hemozoin and also were shown to have an elevated ZnPPIX/FePPIX ratio like trophozoites. The demarcation to trophozoite stage is the point that hemozoin is visible under oil immersion microscopy, but 3 to $12 \mathrm{~h}$ before that point, heme crystals are still made $(2,39)$.

When ZnPPIX or the quinoline/FePPIX complex binds on a point of FePPIX crystal, extension could proceed adjacent to the site of inhibitor binding (17). ZnPPIX may need to reach more than 10 times the in vitro $\mathrm{IC}_{50}$ to have an inhibitory effect by surface binding. Because ZnPPIX, like FePPIX, readily absorbs proteins nonspecifically, a higher concentration would be necessary to inhibit large crystal formation. If the $\pi-\pi$ interactions of ZnPPIX with FePPIX strictly acted by prevention of substrate from incorporation into formation of crystal, one should observe no increase in the ratios of ZnPPIX to FePPIX from purified FePPIX crystals. Bohle and colleagues have powder diffraction data for a head to tail heme crystal dimer rather than a heme polymer (4). Even though the reactive iron is no longer able to participate in Fenton chemistry to make oxygen radicals once the coordinate bond is made, the head-to-tail dimer may still be able to intercalculate in membranes like heme monomer. Formation of the large hemozoin crystals, targeted by the quinolines and ZnPPIX, is then postulated to be a means of preventing membrane toxicity of the crystal dimers.

The $\mathrm{IC}_{50}$ of $5 \mu \mathrm{M}$ is substantially less than the $500 \mu \mathrm{M}$ previously reported by Martiney and Cerami or the $1.1 \mathrm{mM}$ reported by Cole and Wright. However the assay used by Martiney uses approximately $125 \mathrm{nmol}$ of hemozoin crystals and $15 \mu \mathrm{mol}$ of FePPIX substrate (26). The assay may be described in a sense as a radioactive FePPIX binding assay with minimal extension. Chloroquine in this assay has an $\mathrm{IC}_{50}$ of $120 \mu \mathrm{M}$. The Cole/Wright assay mirrored that of Egan who reported a $6 \mathrm{mM} \mathrm{IC}_{50}$ for chloroquine. This assay uses a very high insoluble concentration of $2.2 \mathrm{mM}$ FePPIX at $60^{\circ} \mathrm{C}$ and in a sense is an acid precipitation assay performed at the high temperature of $60^{\circ} \mathrm{C}$. The $\mathrm{IC}_{50}$ of $5 \mu \mathrm{M}$ reported here in this assay with $50 \mu \mathrm{M}$ FePPIX is close to that noted for chloroquine and quinidine (15). In all the different assays, the IC of ZnPPIX is close to chloroquine.

The results that show minimal inhibition of crystal extension in vitro with prebound ZnPPIX, chloroquine, or quinidine are explained by a mixture of covalent and noncovalent interactions for ZnPPIX and the noncovalent interactions for chloroquine and quinidine. The washes or dilution allows for a dissociation of noncovalent inhibitor, which then permits FePPIX extension to occur at a few sites. At equilibrium without washes or dilution of inhibitor association and disassociation in the test tube or parasite digestive vacuole inhibition of heme crystallization is achieved.

Normal erythrocytes have a 1:40000 ratio of ZnPPIX to FePPIX from hemoglobin equal to $25 \mu \mathrm{mol} Z$ nPPIX:1 mol FePPIX. In Plasmodium, ZnPPIX accumulates in the parasite 10-fold more than in normal erythrocytes to 1:4000 or $250 \mu \mathrm{mol} Z$ nPPIX to 1 mol FePPIX. In iron deficiency, the starting concentration of ZnPPIX in the erythrocyte is up 10-fold to $5 \mu \mathrm{M}$, which also equals to 1:4000 or $250 \mu \mathrm{mol} Z \mathrm{ZnPPIX}$ to $1 \mathrm{~mol}$ of FePPIX. Theoretically, in the erythrocyte of iron deficiency, the Plasmodium parasite may then encounter 100-fold concentration increases in ZnPPIX to $50 \mu \mathrm{M}$ or 1:400, which would also equal $2500 \mu \mathrm{mol} Z$ nPPIX to 1 mol FePPIX. This concentration is sufficient for saturable binding and inhibition of FePPIX crystal formation in vitro. Unique to Plasmodium infection, the normal erythrocyte has a potential drug, ZnPPIX, already present in the host cell cytosol at $0.5 \mu \mathrm{M}$. Alteration in the erythrocyte environment increases this potential drug 10- to 100 -fold in the case of iron deficiency. ZnPPIX is delivered along with hemoglobin to the site of action of the quinolines in the digestive vacuole of the Plasmodium parasite. The ZnPPIX concentration increase should be regarded as an average for millions of heterogenous erythrocytes counted. A small portion of the erythrocytes will have a lower concentration of ZnPPIX that is capable of supporting Plasmodium multiplication in the face of a high average concentration of ZnPPIX. The moderate elevation of ZnPPIX associated with anemia of chronic disease or acute infection is most likely not high enough to exert a significant effect on the inhibition of the parasite.

The implications for public health are that only the severely anemic individuals may have an intrinsic mechanism of protection from very high parasitemias. The ZnPPIX:FePPIX ratio is the average of a population of erythrocytes and still may support parasitemia. Most moderate and some severely anemic individuals should not have a ZnPPIX to FePPIX ratio above 250 and would not have exacerbation of malaria with iron replenishment therapy. The authors strongly support iron supplementation for the positive growth and cognitive benefits (24). Iron supplementation should not be withheld for fear of exacerbating malaria. Rather, severely anemic individuals with elevated ZnPPIX:FePPIX (above 250) should be targeted for antimalarial chemoprophylaxis. Further epidemiological studies designed specifically to this question of protection from severe malaria disease are a next step. 


\section{ACKNOWLEDGMENTS}

DJS received financial assistance from the Pew Scholars Program in Biomedical Sciences and NIH ROI A145774. A NCCR Grant GPDGCRC RR0052 supports the use of RBC for culturing P. falciparum. The authors are indebted to Bradley Harris, Carole Cooke, and Michael Delannoy in the Johns Hopkins University Dept. of Cell Biology and Anatomy for assistance with FEISEM and acknowledge Rebecca Stoltzfus for helpful discussions.

Address correspondence and reprint requests to David J Sullivan, Department of Molecular Microbiology and Immunology, School of Hygiene and Public Health, Johns Hopkins University, 615 N. Wolfe Street, Baltimore, MD 21205. Phone: 410-614-1562; fax: 410-955-0105; e-mail: dsulliva@jhsph.edu.

Submitted April 9, 2003; accepted for publication September 3, 2003.

\section{REFERENCES}

1. Francis SE, Sullivan Jr DJ, Goldberg DE. (1997) Hemoglobin metabolism in the malaria parasite Plasmodium falciparum. Annual Rev. Microbiol. 51:97-123.

2. Krugliak M, Zhang J, Ginsburg H. (2002) Intraerythrocytic Plasmodium falciparum utilizes only a fraction of the amino acids derived from the digestion of host cell cytosol for the biosynthesis of its proteins. Mol. Biochem. Parasitol. 119:249-56.

3. Slater AF et al. (1991) An iron-carboxylate bond links the heme units of malaria pigment. Proc. Natl. Acad. Sci. U.S.A. 88:325-9

4. Pagola S, Stephens PW, Bohle DS, Kosar AD, Madsen SK. (2000) The structure of malaria pigment beta-hematin. Nature 404:307-10.

5. Slater AF, Cerami A. (1992) Inhibition by chloroquine of a novel haem polymerase enzyme activity in malaria trophozoites. Nature 355:167-9.

6. Sullivan D. (2002) Hemozoin: a biocrystal synthesized during the degradation of hemoglobin. In: Matsumura S, Steinbüchel A (eds.) Miscellaneous Biopolymers, Biodegradation of Synthetic Polymers. Wiley-VCH Verlag GmbH \& Co, Weinheim, Germany, pp. 129-63.

7. Sullivan DJ. (2002) Theories on malarial pigment formation and quinoline action. Int. J. Parasitol. 32:1645-53.

8. Slater AF. (1993) Chloroquine: mechanism of drug action and resistance in Plasmodium falciparum. Pharmacol. Ther. 57:203-35.

9. Ginsburg H, Ward S, Bray P. (1999) An integrated model of chloroquine action. Parasitol. Today 15:357-60.

10. Cohen SN, Phifer KO, Yeilding KL. (1964) Complex formation between chloroquine and ferrihaemic acid in vitro, and its effect on the antimalarial action of chloroquine. Nature 202:805-6.

11. Chou AC, Chevli R, Fitch CD. (1980) Ferriprotoporphyrin IX fulfills the criteria for identification as the chloroquine receptor of malaria parasites. Biochem. 19:1543-9.

12. Moreau S, Perly B, Chachaty C, Deleuze C. (1985) A nuclear magnetic resonance study of the interactions of antimalarial drugs with porphyrins. Biochem. Biophys. Acta 840:107-16.

13. Vippagunta SR et al. (1999) Structural specificity of chloroquine-hematin binding related to inhibition of hematin polymerization and parasite growth. J. Med. Chem. 42:4630-9.
14. Leed A, DuBay K, Ursos LM Sears D De Dios AC, Roepe PD. (2002) Solution structures of antimalarial drug-heme complexes. Biochem. 41:10245-55.

15. Sullivan DJ, Jr., Gluzman IY, Russell DG, Goldberg DE. (1996) On the molecular mechanism of chloroquine's antimalarial action. Proc. Natl. Acad. Sci. U.S.A. 93:1 1865-70.

16. Sullivan Jr. DJ, Matile H, Ridley RG, Goldberg DE. (1998) A common mechanism for blockade of heme polymerization by antimalarial quinolines. J. Biol. Chem. 273:31 103-7.

17. Buller R, Peterson ML, Almarsson O, Leiserowitz L. (2002) Quinoline binding site on malaria pigment crystal: a rational pathway for antimalaria drug design. Crystal Growth Design 2:553-62.

18. Feldman F, Lichtman HC. (1964) In vitro porphobilinogen and porphyrin synthesis in thalassemia major and sickle cell anemia. Ann. N.Y. Acad. Sci. 119:540-7.

19. Lamola AA, Yamane T. (1974) Zinc protoporphyrin in the erythrocytes of patients with lead intoxication and iron deficiency anemia. Science 186:936-8.

20. Hastka J, Lasserre J, Schwarzbeck A, Strauch M, Hehlmann R. (1993) Zinc protoporphyrin in anemia of chronic disorders. Blood 81:1200-4.

21. Stoltzfus RJ, Chwaya HM, Montresor A, Albonico M, Savioli L, Tielsch JM. (2000) Malaria, hookworms and recent fever are related to anemia and iron status indicators in 0- to 5-y old Zanzibari children and these relationships change with age. J. Nutr. 130:1724-33.

22. Yip R. (1998) Iron deficiency. Bull. WHO 76:121-3.

23. Stoltzfus RJ, Chwaya HM, Albonico M, Schulze K, Savioli L, Tielsch JM. (1996) Serum ferritin, erythrocyte protoporphyrin and hemoglobin are valid indicators of iron status of school children in a malaria-holoendemic population. J. Nutr. $127(2): 293-8$.

24. Shankar AH. (2000) Nutritional modulation of malaria morbidity and mortality. J. Infect. Dis. 182 Suppl 1:\$37-53.

25. Williams T et al. (1996) High incidence of malaria in alpha-thalassemic children Nature 383:522-5.

26. Martiney JA, Cerami A, Slater AF. (1996) Inhibition of hemozoin formation in Plasmodium falciparum trophozoite extracts by heme analogs: possible implication in the resistance to malaria conferred by the beta-thalassemia trait. Mol. Med. 2:236-46.

27. Basilico N, Monti D, Olliaro P, Taramelli D. (1997) Non-iron porphyrins inhibit $\beta$-haematin (malaria pigment) polymerisation. FEBS Lett. 409:297-9.

28. Cole KA, Zeigler J, Evans CA, Wright DW. (2000) Metalloporphyrins inhibi $\beta$-hematin (hemozoin) formation. J. Inorg. Biochem. 78:109-15.

29. Egan TJ, Mavuso WW, Ross DC, Marques HM (1997) Thermodynamic factors controlling the interaction of quinoline antimalarial drugs with ferriprotopor phyrin IX. J. Inorg. Biochem. 68:137-45.

30. Chisolm JJ, Brown D. (1975) Micro-scale photofluorometric determination of "free erythrocytic porphyrin" (Protoporphyrin IX). Clin. Chem. 21 (11):1669-77.

31. Fitch C, Cai G, Chen Y, Shoemaker J. (1999) Involvement of lipids in ferriprotoporphyrin IX polymerization in malaria. Biochim. Biophys. Acta 1454:31-7.

32. Trager W, Jensen JB. (1976) Human malaria parasites in continuous culture. Science 193:673-5.

33. Lambros C, Vanderberg JP. (1979) Synchronization of Plasmodium falciparum erythrocytic stages in culture. J. Parasitol. 65:418-20.

34. Christophers SR, Fulton JD. (1939) Experiments with isolated malaria parasites (Plasmodium knowlesi) free from red cells. Ann. Trop. Med. Parasit. 33:161.

35. Chen MM, Shi L, Sullivan DJ. (2001) Haemoproteus and Schistosoma synthesize heme polymers similar to Plasmodium hemozoin and $\beta$-hematin. Mol. Biochem. Parasitol. 113:1-8.

36. Bohle DS, Kosar AD, Stephens PW. (2002) Phase homogeneity and crystal morphology of the malaria pigment $\beta$-hematin. Acta Crystallogr. D. Biol. Crystallogr. 58:1752-6.

37. Noland GS, Briones N, Sullivan DJ. (2003) The shape and size of hemozoin crystals distinguishes diverse plasmodium species. Mol. Biochem. Parasitol. 130:91-9.

38. Eckstein-Ludwig U et al. (2003) Artemisinins target the SERCA of Plasmodium falciparum. Nature 424:957-6

39. Zhang J, Krugliak M. Ginsburg H. (1999) The fate of ferriprotoporphyrin IX in malaria infected erythrocytes in conjunction with the mode of action of antimalarial drugs. Mol. Biochem. Parasit. 99:129-41. 